\title{
Anthracycline-induced cardiotoxicity in the chemotherapy treatment of breast cancer: Preventive strategies and treatment (Review)
}

\author{
FENGFENG CAI $^{1 *}$, MANUEL ANTONIO FALAR LUIS $^{1 *}$, XIAOYAN LIN $^{1 *}$, \\ MINGHONG WANG ${ }^{2,3}, \mathrm{LU} \mathrm{CAI}^{1}, \mathrm{CHUNMEI} \mathrm{CEN}^{1}$ and EWELINA BISKUP ${ }^{4}$
}

\begin{abstract}
Departments of ${ }^{1}$ Breast Surgery and ${ }^{2}$ General Practice, Yangpu Hospital, Tongji University School of Medicine, Shanghai 200090; ${ }^{3}$ Department of Cardiology, Eastern Hepatobiliary Surgery Hospital, Second Military Medical University, Shanghai 200438; ${ }^{4}$ Department of Basic Medical Sciences, Shanghai University of Medicine and Health Sciences, Shanghai 201318, P.R. China
\end{abstract}

Received November 8, 2018; Accepted April 30, 2019

DOI: $10.3892 / \mathrm{mco} .2019 .1854$

\begin{abstract}
Anthracyclines are highly effective chemotherapeutic agents, used for a wide variety of malignancies. Cardiotoxicity is a well-recognized side effect of anthracycline therapy that limits the total amount of drug administered and can cause heart failure in some patients. Most experimental data support oxidative stress as the etiology of anthracycline-induced cardiotoxicity. The objective of this paper was to provide a review of the clinical classification, risk factors, monitoring and prevention of anthracycline-induced cardiotoxicity in patients with breast cancer.
\end{abstract}

\section{Contents}

1. Introduction

2. Mechanism of cardiotoxicity

3. Classification of cardiotoxicity induced by anthracyclines

4. Risk factors for cardiotoxicity induced by anthracyclines

5. Cardiotoxicity monitoring

6. Prevention of cardiotoxicity

7. Oncological strategies to mitigate cardiotoxicity

Correspondence to: Dr Fengfeng Cai, Department of Breast Surgery, Yangpu Hospital, Tongji University School of Medicine, 450 Tengyue Road, Shanghai 200090, P.R. China

E-mail: caifengfeng@tongji.edu.cn

Dr Minghong Wang, Department of General Practice, Yangpu Hospital, Tongji University School of Medicine, 450 Tengyue Road, Shanghai 200090, P.R. China

E-mail: minghongwang2015@163.com

*Contributed equally

Key words: anthracyclines, breast cancer, doxorubicin, cardiotoxicity, risk factors, prevention, treatment
8. Treatment of cardiotoxicity

9. Future directions to avoid cardiotoxicity induced by anthracyclines

10. Conclusion

\section{Introduction}

Breast cancer is one of the most common malignant tumors that endanger the health of women (1), with an estimated 252,710 new cases in the United States in 2017 (2) and 26,000 new cases in Canada (3) in 2016. A total of 1 in 8 women in the United States will have breast cancer in their lifetime (4). Approximately 1.5 million women worldwide develop breast cancer every year, and $\sim 500,000$ women succumb to breast cancer (5). Early detection and advances in screening have led to a 5-year survival rate approaching 90\%, and in the United States, almost 3 million people are living with a prior diagnosis of breast cancer (2). The treatment options usually include a combination of surgery, cytotoxic chemotherapy, radiation therapy and molecularly targeted endocrine therapy, depending on the type of breast cancer diagnosed (6).

At present, anthracyclines and taxanes are the two major classes of drugs for breast cancer treatment. Anthracyclines (Table I) are among the most commonly used and effective drugs in breast cancer treatment. In the past 30 years, they have become an important component of adjunctive and palliative therapy for breast cancer. Anthracyclines belong to a class of antineoplastic antibiotics, which interfere with cell replication by acting on the DNA at several levels, showing an effect in every phase of the cell cycle. Doxorubicin and epirubicin (Fig. 1) are commonly used in clinical practice. Administration is only via an intravenous infusion; metabolism is hepatic and excretion via the bile route, while urinary elimination accounts for approximately $1 / 6$ of the total amount. Although anthracyclines exhibit a range of toxic effects, including transient myelosuppression, mucositis and hair loss, cardiotoxicity still remains a prominent risk since it may be permanent and progressive, leading to multimorbidity and severely impacting quality of 
life in patients with breast cancer. Acute cardiotoxicities, as well as the potential effect of cumulative doses, increasing the risk of congestive heart failure, are crucial and should be considered when deciding on a treatment strategy. The present study presents a concise review of the literature, focusing on anthracycline-induced cardiotoxicity, its pathophysiology, prevention, monitoring and outcomes. A comprehensive literature review has been conducted. A bibliographic search was performed in the Cochrane, Medline, PubMed, Scopus, Web of Science and Scielo databases. Databases were searched systematically using the following key words: Anthracyclines, breast cancer, risk factors, prevention and treatment, combined with cardiotoxicity, cardiomyopathy or heart failure.

\section{Mechanism of cardiotoxicity}

The pathophysiology of cardiotoxicity induced by anthracyclines has not been fully elucidated. Until recently, the most widely accepted hypothesis was that cardiotoxicity by anthracycline is due to reactive oxygen species (ROS) generated by the quinone moiety common to all anthracyclines. However, a recent study by Zhang et al (7) supports an alternative model, reporting that the toxicity is caused as anthracyclines block the function of topoisomerase II $\beta$ (TopIIB) causing double-stranded DNA breaks, which in turn leads to activation of p53 tumor-suppressor protein, mitochondrial dysfunction, and the generation of ROS that result in cardiac cell death.

Role of reactive oxygen species. The pathological changes include cardiomyocyte apoptosis or necrosis, loss of myofibrils, expansion of the sarcoplasmic reticulum and mitochondrial swelling (8). Those can be explained by the mechanism of action of anthracyclines. In the process of metabolism, the semiquinone radical of the C-ring is reduced by several single electron oxidoreductases to form a semiquinone free radical. Semiquinone free radicals react with oxygen to produce superoxide anions, and the latter can disproportionate into hydrogen peroxide. Doxorubicin can also bind to nitrous oxide synthase, which leads to reactive nitrogen, particularly the production of peroxy-nitrite. In addition, anthracycline antitumor drugs can also generate free radicals through non-enzymatic pathways. These reactive oxygen species and reactive nitrogen can cause mitochondrial functional damage, energy imbalances, even cardiomyocyte apoptosis. This is currently the most studied and most widely accepted mechanism of myocardial injury, but the clinical application of antioxidants to prevent anthracycline cardiotoxicity has not achieved the desired results, putting the 'reactive oxygen and oxidative stress theory' into question (9).

Some studies also suggest the doxorubicin-induced cardiotoxicity is mediated by the doxorubicinol (DOXol) metabolite. A study found that the anthracycline side chain C-13 can be reduced to doxorubicin doxorubicinol (DOXol) by NADPH-dependent reductase in vivo (10). The metabolite still retains cytotoxicity, but can affect energy metabolism in the heart muscle, changes in ion concentrations and $\mathrm{Ca}^{2+}$ transport, ultimately leading to a decrease in myocardial contractile function, and therefore DOXol is thought to be involved in an anthracycline-type cardiotoxicity mechanism. Animal experiments found that DOXol selectively accumulates in the heart. At autopsy, in patients treated with doxorubicin, high concentrations of DOXol may be found in cardiomyocytes (11). Mordente et al (12) also considered that secondary alcohol metabolites may play an important role in the development of anthracycline-induced congestive heart failure and end-stage cardiomyopathy, and is one of the pathogenic factors of anthracycline-type cardiotoxicity (Fig. 2). Secondary metabolites are slightly less active at redox cycling, but markedly more potent at dysregulating calcium and iron homeostasis. They also produce oxidative stress, ion dysregulation, and concomitant alterations of cardiac-specific gene expression, eventually inducing cardiomyopathy.

Some studies also suggest that anticancer drugs, such as anthracycline, induce cardiotoxicity mediated by the hERG channel, which is one of several potassium-selective voltage-gated channels that participate in the control of the electrical activity of the human heart $(13,14)$. Systemic treatment of cancers with hERG antagonists may affect cardiac myocytes, resulting in apoptosis and heart failure (15). Due to its crucial role in the cardiac action potential, impairment of hERG channel function can lead to severe cardiac disorders, manifested by altered QT intervals (13). For instance, inherited loss-of-function mutations in hERG can cause long QT syndrome, which predisposes individuals to life-threatening torsades de pointes arrhythmia (16).

\section{Classification of cardiotoxicity induced by anthracyclines}

The cardiotoxicity induced by these drugs can be classified as acute, subacute and chronic, which can be further categorized into type I (early onset) and type II (late onset) $(17,18)$.

Acute toxicity is rare and reversible, occurring in the course of administration or within 1 week after administration as transient arrhythmia, e.g. supraventricular tachycardia, nonspecific ST segment or T wave abnormality, pericardial myocarditis syndrome or acute left ventricular failure. The subacute cardiotoxicity can occur several days to several weeks after administration, manifesting as acute left heart failure, myocarditis, and pericarditis. Type I chronic cardiotoxicity manifests at least one year after the completion of chemotherapy, mostly years decades after the chemotherapy, mainly as occult ventricular dysfunction, congestive heart failure, and arrhythmia. Type II chronic cardiotoxicity is typically caused by novel biological-targeted antibodies $(9,19)$.

\section{Risk factors for cardiotoxicity induced by anthracyclines}

Cumulative dose. The total cumulative dose of anthracyclines is the most significant risk factor for cardiac dysfunction (20). There is a clear relationship between the occurrence of anthracycline cardiotoxicity and the cumulative dose of the drug. Von Hoff et al (21), in a retrospective analysis, found that when a patient receives a cumulative dose of doxorubicin at 400,550 and $700 \mathrm{mg} / \mathrm{m}^{2}$, the incidence of cardiotoxicity is 3,7 and $18 \%$, respectively, with dose-limiting toxicity. Therefore, it is recommended that the cumulative dose of doxorubicin should not exceed $550 \mathrm{mg} / \mathrm{m}^{2}$. If treated with epirubicin, the cumulative dose recommendation is to not exceed $900 \mathrm{mg} / \mathrm{m}^{2}$. Ryberg et al (22) performed a risk analysis of 1,097 patients with metastatic breast cancer who 
Table I. Commonly used chemotherapeutics in the treatment of breast cancer.

$\begin{array}{ll}\text { Cancer treatment } & \text { Cardiovascular adverse effects }\end{array}$

Anthracyclines (eg, epirubicin, doxorubicin, daunorubicin, idarubicin,)

Taxanes (e.g., paclitaxel)

Alkylating agents (e.g., cyclophosphamide, cisplatin, mitomycin, ciplastin, ifosfamide)

Endocrine therapy (eg, anastrozole, letrozole, tamoxifen)

Cyclin-dependent kinase 4/6 inhibitors (e.g., ribociclib, palbociclib, lapatinib, sorafenib, lapatinib)

Antimetabolites (e.g., capecitabine, 5-fluorouracil, cytarabine, methotrexate)

HER-2-directed therapies (e.g., pertuzumab, trastuzumab) Radiation therapy
Ventricular fibrillation, myocarditis, heart failure, ventricular tachycardia, left ventricular dysfunction, pericarditis, atrial fibrillation

Ventricular ectopy, bradycardia, heart block

Heart failure, heart block, supraventricular tachycardia, congestive heart failure, left ventricular dysfunction, bradycardia, atrial fibrillation, arterial thrombosis, myocarditis, pericarditis Thromboembolism, valvular dysfunction, venous thrombosis, heart failure, pericarditis, peripheral atherosclerosis, dysrhythmia QTc prolongation

Congestive heart failure, ventricular tachycardia, coronary artery spasm, ventricular fibrillation, coronary thrombosis, atrial fibrillation

Heart failure, left ventricular dysfunction,

Cardiomyopathy, coronary artery disease, valvular disease pericar dial disease, arrhythmias

Modified from (1).<smiles>COc1cccc2c1C(=O)c1c(O)c3c(c(O)c1C2=O)C[C@@](O)(C(=O)CO)C[C@@H]3O[C@H]1C[C@H](N)[C@@H](O)[C@H](C)O1</smiles>

Doxorubicin<smiles>COc1cccc2c1C(=O)c1c(O)c3c(c(O)c1C2=O)C[C@@](O)(C(=O)CO)C[C@@H]3O[C@H]1C[C@@H](N)[C@H](O)[C@H](C)O1</smiles>

Epirubicin

Figure 1. Structures of the anthracyclines currently used.

had previously been treated with epirubicin; the maximum cumulative dose for epirubicin treatment was determined according to the patient's risk level, to keep the incidence of congestive heart failure below $5 \%$. The results showed that for patients at an average age of 40, without other risk factors for congestive heart failure, the recommended cumulative dose is $806 \mathrm{mg} / \mathrm{m}^{2}$, and an average age of 70 , the maximum cumulative dose is $609 \mathrm{mg} / \mathrm{m}^{2}$.

Age. Von Hoff et al (21) showed that the occurrence of anthracycline cardiac toxicity was significantly associated with age. The study included 4,018 patients with a median age of 49, and the incidence of anthracycline cardiotoxicity increased steadily with age $(\mathrm{P}=0.0002)$. A retrospective analysis by Swain et al (23) showed that when the cumulative dosage of doxorubicin reached $400 \mathrm{mg} / \mathrm{m}^{2}$, the risk of congestive heart failure in patients over 65 years old was 2.25 times higher than that in patients under 65 years old.
For patients of age $>65$ years, the recommended dosage ratio of dexrazoxane: Doxorubicin is 10:1; doxorubicin should be given within $30 \mathrm{~min}$ of giving dexrazoxane.

Existing cardiac risk factors. Patients with preexisting cardiac risk factors have an increased incidence of congestive heart failure when treated with anthracyclines (24). Studies by Ryberg et al (22) showed that patients with cardiac risk factors (such as hypertension, diabetes, obesity, hyperthyroidism and obstructive lung disease) had a 3-times greater incidence of cardiac toxicity and were not affected by the cumulative dose.

Dose reduction reduces the incidence of early myocardial dysfunction, but does not remove the risk of long-term problems. A study reported reported that continuous infusions of free doxorubicin between 48 and $96 \mathrm{~h}$ reduce cardiotoxicity (25). Retrospective analyses have found that weekly doses of doxorubicin are considerably less cardiotoxic than administering the drug every 3 weeks. 


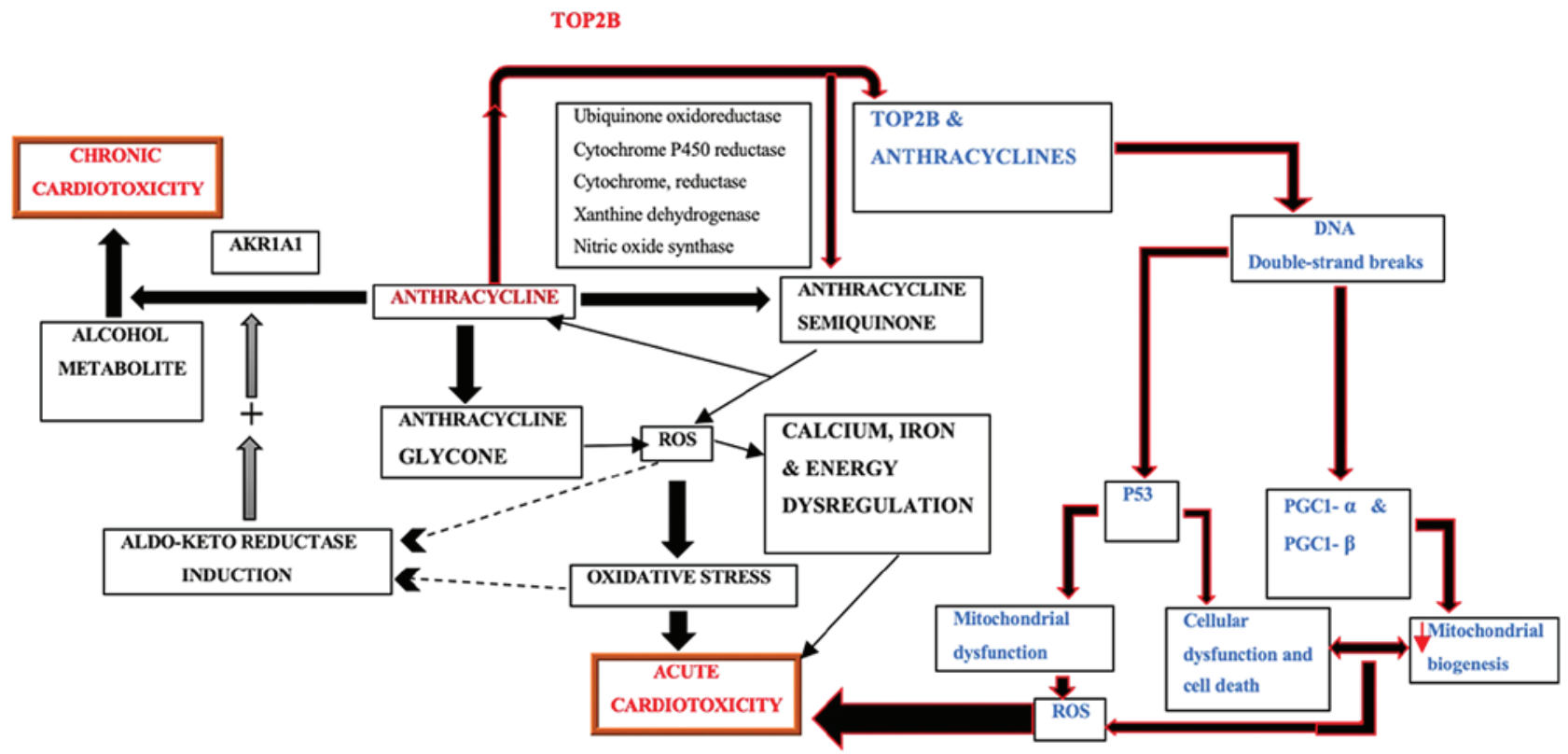

Figure 2. Unified mechanism for anthracycline-induced cardiotoxicity. ROS, reactive oxygen species; TOP2B, DNA topoisomerase II $\beta$; PGC, PPARG coactivator 1.

Radiotherapy. Cardiac radiation exposure markedly increases myocardial sensitivity to anthracyclines. Concomitant exposure to cyclophosphamide, bleomycin, vincristine, amsacrine or mitoxantrone may predispose to cardiotoxicity (26). Black ethnic origin (26) and trisomy 21 (26) increase the risk of early cardiotoxicity.

An increased incidence of cardiac failure and severe cardiac abnormalities more frequently occur at longer follow-up (27).

\section{Cardiotoxicity monitoring}

Electrocardiogram (ECG). Electrocardiogram changes occur earlier, and are non-specific, transient and reversible. The most common ECG abnormalities are ST- and T-wave changes (Fig. 3), sinus tachycardia, QT prolongation, ventricular premature contractions and transient atrial contractions. A study showed that prolongation of the QTc interval predicts a high risk of developing malignant ventricular arrhythmias (28). ECG is a simple, convenient and non-invasive method, commonly used for the detection of cardiotoxicity in clinical practice. However, because it is affected by many factors, the detection value is not large.

Echocardiography. Echocardiography is a common method for clinical diagnosis of heart failure, mainly used to detect the left ventricular ejection fraction (LVEF), and is widely used in clinical practice because of its non-invasiveness. However, abnormal changes in left ventricular systolic function or changes in the morphological structure can only occur when the myocardium is severely damaged or the overall function of the heart is impaired (29), and the examination results are greatly affected by the experience of the sonographer, which is not conducive to the early detection of lesions. In the present context, anthracyclines are cardiotoxic and it is necessary to evaluate the LVEF of a patient every 3 months. If a patient develops clinical symptoms of cardiotoxicity during anthracycline treatment, or are asymptomatic with LVEF $<45 \%$ or a decrease to the baseline of $15 \%$, then the treatment should be discontinued to adequately assess the patient's cardiac function, and further treatment should be administered with caution.

Tissue doppler imaging (TDI). Tissue doppler imaging (TDI) is an application of the Doppler effect, based on the traditional color Doppler technique, for the detection of ventricular muscle, mitral systole and diastolic frequency, providing ultrasound imaging information about myocardial motion. Compared with traditional Doppler, the influence of the cardiac load state is reduced and the result is more reliable. In a study by Tassan-Mangina et al (30), TDI was able to detect left cardiac early diastolic and late systolic functional impairment through follow-up of patients treated with medium-dose anthracycline. If the isovolumic contraction time at the mitral valve is less than $80 \mathrm{msec}$, it may indicate late damage to ventricular function. Jurcut et al (31) studied 16 elderly patients with breast cancer who were treated with liposomal doxorubicin, and found that the longitudinal and radial deformations of ventricular systolic phase identified by Doppler myocardial imaging were comparable to those found using traditional echocardiography. The strain rate decreased significantly and, in particular, the radial functional change was more pronounced and minor lesions to the myocardium were effectively detected.

Radionuclide angiocardiography. Also known as gated multiple gated acquisition (MUGA), this is a widely used method for detecting the ejection fraction (21). Mitani et al (32) found in a retrospective analysis that, during doxorubicin therapy, using radionuclide continuous equilibrium monitoring for early detection of the decrease in LVEF is an effective and economical way of preventing the development of congestive heart failure. This method has high reproducibility and can reliably monitor the decrease in LVEF. However, the accumulation of radiation exposure limits its regular use. This method 


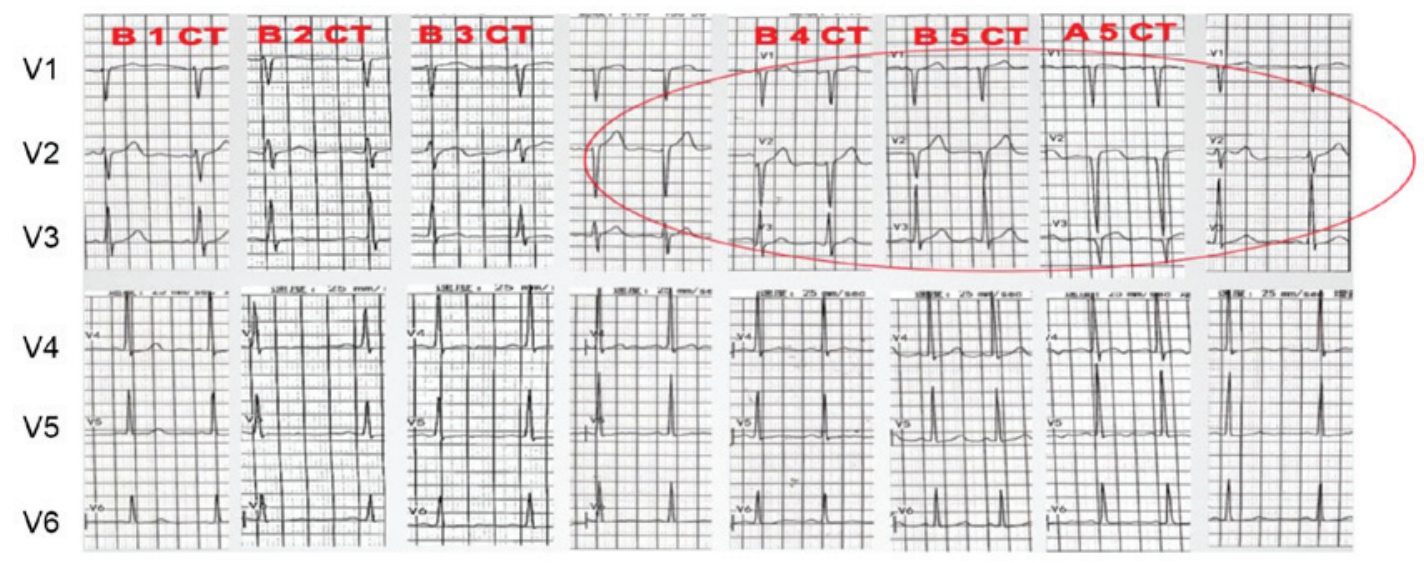

Figure 3. Comparative electrocardiogram data of a 55-year-old female who had reversible cardiotoxicity due to chemotherapy with trastuzumab. In V1-3, the R-wave disappeared, and the V4-6 T wave was low and flat. B, before; A, after; 1 CT, first round of chemotherapy; 2 CT, second round of chemotherapy; 3 CT, third round of chemotherapy; $4 \mathrm{CT}$, fourth round of chemotherapy; $5 \mathrm{CT}$, fifth round of chemotherapy.

is a recommended option when patient treatment or a clinical trial requires repeated precise testing (33).

Magnetic resonance imaging (MRI). Cardiac magnetic resonance (CMR) can evaluate heart morphology, tissue function, metabolism and blood flow changes, can show the location and extent of myocardial infarction-related edema and necrosis, and is not affected by cardiac function. Although CMR is an ideal method for detecting myocardial function and its damage, repeated examinations are expensive and it is not the preferred or a routine measure (34).

Endomyocardial biopsy. Cardiac biopsy is currently the most sensitive indicator of chronic cardiotoxicity induced by anthracycline treatment (35), but anthracycline-induced myocardial injury generally presents as scattered or left ventricular lesions. The majority of biopsy samples are taken from the right ventricle, which may underestimate the severity of the lesion. However, cardiac biopsy is not routinely performed in current practice because of its invasiveness, so it should not be used as a routine choice in early monitoring.

\section{Serum biochemical indicators}

Cardiac troponin. Cardiac troponin (cTn) is a polypeptide subunit of the troponin complex. Troponin is an important regulatory protein involved in muscle contraction and consists of three subunits: Cardiac troponin C (cTnC), cardiac troponin $\mathrm{T}(\mathrm{cTnT})$, and cardiac troponin (cTnI). When degeneration and necrosis occur in the myocardium, cTnI and cTnT enter the peripheral blood. Several studies $(36,37)$ have reported increased levels of cTnT/TnI in patients treated with anthracycline chemotherapy, associated with cardiac diastolic dysfunction. cTnT/TnI can detect early cardiotoxicity caused by anthracyclines such as ADM before significant LVEF changes occur (38). However, cardiac troponin lacks specificity in the assessment of cardiotoxicity caused by anthracycline chemotherapy, and it is necessary to combine other auxiliary examinations for comprehensive analysis and diagnosis.

$B$-type brain natriuretic peptide (BNP) and $N$-terminal pro-brain natriuretic peptide (NT-proBNP). In recent years,
BNP and NT-proBNP have garnered attention in the diagnosis of early cardiac toxicity and have been gradually applied in clinical practice. A rapid and accurate indicator of heart failure caused by anthracyclines, BNP contains 32 amino acids, with a signal peptide of 26 amino acids, and has a half-life of 15 to 2 min. NT-proBNP contains 76 amino acids with a half-life of 60-120 min (39). Because NT-proBNP has a long half-life and is stable, it can accumulate to higher concentrations, so it can diagnose mild systolic or diastolic heart failure and asymptomatic left ventricular dysfunction. Zhang et al (40) monitored NT-proBNP in 50 patients with breast cancer using anthracyclines and found that the value of NT-proBNP gradually increased with the progress of chemotherapy, before chemotherapy and after the first course of chemotherapy.

\section{Prevention of cardiotoxicity}

Limiting cumulative dose. Maximum doses of anthracyclines have been implemented. Maximum cumulative doses of $400-550 \mathrm{mg} / \mathrm{m}^{2}$ doxorubicin and $900 \mathrm{mg} / \mathrm{m}^{2}$ epirubicin are currently recommended $(41,42)$.

Prolonging administration time. Cardiac toxicity can be reduced by weekly low doses and a prolonged continuous infusion time (24-96 h). An analysis showed that slow intravenous infusion of doxorubicin ( $>6 \mathrm{~h}$ ) over a prolonged period could reduce the risk of clinical heart failure and subclinical myocardial injury (43). The cardiotoxicity of the weekly treatment regimen was less than that of the usual 3-week treatment regimen (0.8 vs. $2.9 \%)$ (44).

Strengthening the monitoring of cardiotoxicity. During the use of anthracycline drugs, the monitoring of cardiotoxicity should be strengthened to achieve early detection and early treatment, to prevent the occurrence of severe cardiotoxicity. There are currently various reported methods of monitoring cardiac toxicity: Cardiac endocardium biopsy, electrocardiogram, echocardiography, myocardial backscatter integration parameters, tissue Doppler imaging, ischemic modified albumin, troponin, brain natriuretic peptide and the Tei index. These monitoring methods have certain limitations, such as 

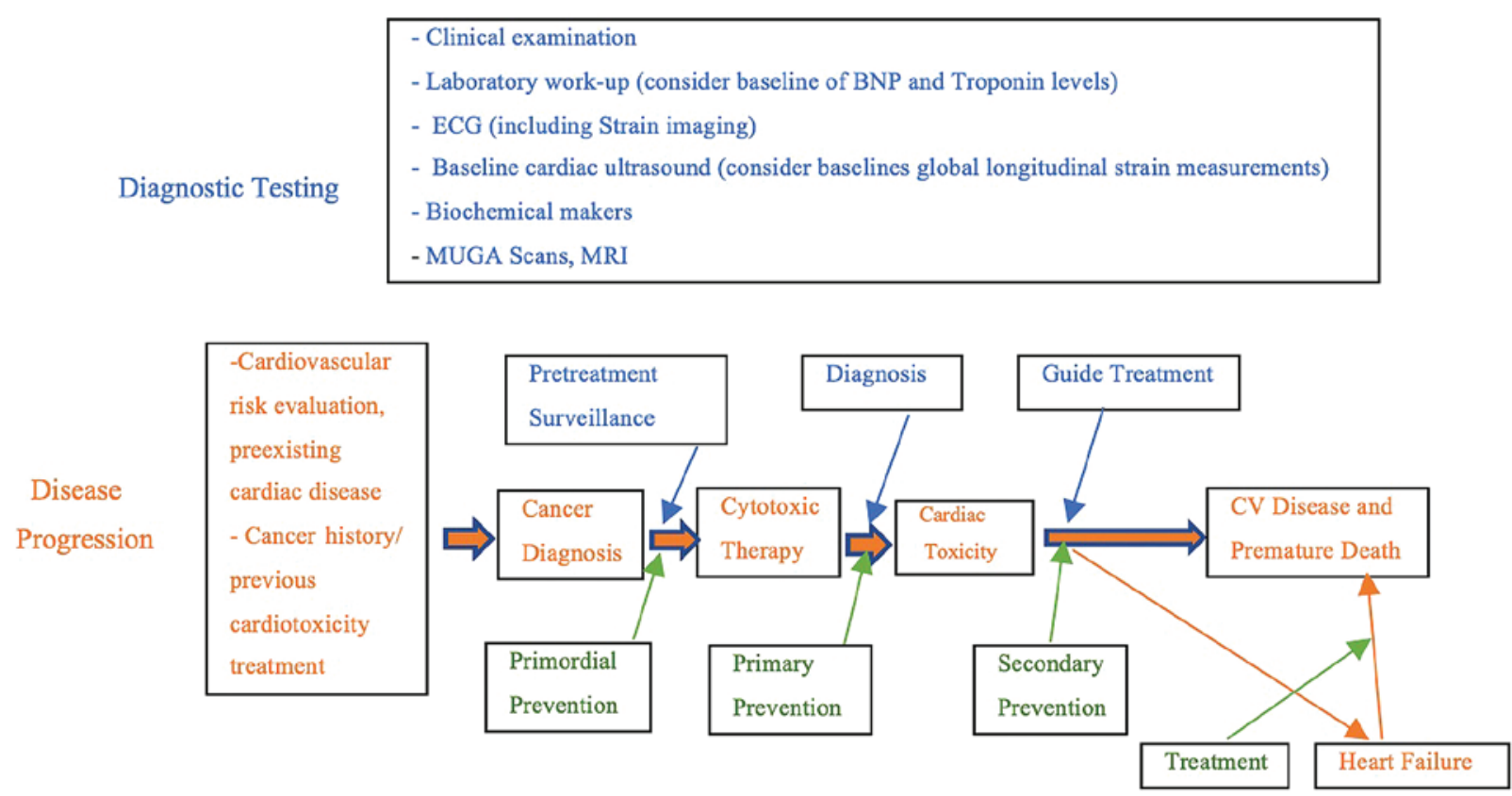

\begin{tabular}{l|l|} 
& - Converting enzyme inhibitors ACEI \\
Treatment & - Diuretics \\
& - Beta blockers \\
\hline
\end{tabular}

Figure 4. Simplified depiction of the detection and treatment of cardiotoxicity in breast cancer. Schematic of the continuum of breast cancer treatment in relation to subsequent cardiotoxicity and heart failure. Modified from Khouri et al (60). BNP, B-type natriuretic peptide; ECG, electrocardiogram; CV, cardiovascular; MUGA, multigated acquisition; MRI, magnetic resonance imaging.

invasiveness and low specificity (45). Therefore, continuous efforts are needed to find sensitive, specific and non-invasive monitoring techniques and tools.

Development of liposome anthracyclines. Liposome anthracycline is used to encapsulate the drug in a lipid body to protect the drug from degrading and inactivating in the plasma, and makes use of structural defects, such as vascular endothelial discontinuity and lymphatic vessel breakage in the tumor tissue, so that the anthracycline drugs selectively penetrate the vascular system and enter the tumor tissue. This reduces the concentration of the drug in the normal endothelial tissue and can significantly reduce the toxicity to the heart, while increasing the anti-tumor index and expanding the antitumor spectrum. Single-use liposome in the treatment of metastatic breast cancer has the same curative effect as doxorubicin and the incidence of cardiac toxicity is significantly lower (13 vs. $29 \%$ ), the cardiac toxicity occurs later and the cumulative dose is larger (46).

\section{Oncological strategies to mitigate cardiotoxicity}

Iron chelating agents. Dexrazoxane (DEX), a most promising cardioprotective agent, has been shown to be effective in reducing both acute and chronic cardiotoxicity induced by anthracycline therapy (47). It has been widely used in the United States and Europe for various clinical applications. Dexrazoxane is mainly used to reduce the incidence and severity of cardiomyopathy caused by doxorubicin in patients with advanced breast cancer. It is applicable to patients who receive a dose of $300 \mathrm{mg} / \mathrm{m}^{2}$ of doxorubicin and need to continue to use doxorubicin. It is only drug approved by the US FDA to protect and reduce the cardiotoxicity of anthracyclines. Multicenter randomized controlled clinical trials have shown that dexrazoxane has a significant cardioprotective effect in breast cancer patients receiving anthracycline chemotherapy, and does not affect the antitumor efficacy of anthracyclines (48). Its protection mechanism is mainly through reducing the production of oxygen free radicals, lipid peroxidation products and myocardial apoptosis (49).

A multicenter phase II clinical trial in China also confirmed that dexrazoxane combined with doxorubicin in the treatment of breast cancer and lymphoma has a significant protective effect on cardiotoxicity caused by doxorubicin. At the same time, dexrazoxane not only has a certain protective effect on the occurrence of cardiotoxicity, but also has a certain reparative effect on damage already done (50). The 2011 edition of the Chinese Expert Consensus on the Prevention of Cardiotoxicity of Anthracycline Anticancer Drugs (51) has also indicated that dexrazoxane has a cardioprotective effect on patients receiving anthracycline chemotherapy.

Some authors have suggested that repeated imaging can be used to detect and assess treatment with iron chelating agents, especially echocardiography and CMR.

Angiotensin-converting enzyme inhibitors (ACEI). These drugs also reduce the cardiotoxicity of anthracyclines (11). In an early study, it was concluded that zofenopril has a complete protective effect on cardiomyocytes from chronic cardiomyopathy caused by doxorubicin, without affecting the 
antitumor activity of doxorubicin (52). A recent rat experiment also demonstrated that low-dose and high-dose zofenopril had a complete protective effect against electrophysiological changes (QT interval prolongation) caused by long-term use of doxorubicin (1.5 mg/kg qwx5) (53).

$\beta$-blockers. Carvedilol is used to treat symptomatic congestive heart failure, reducing the mortality and hospitalization rates of cardiovascular disease. Kalay et al (54) demonstrated in a prospective study that prophylactic use of carvedilol in the treatment of breast cancer patients treated with anthracyclines protects left ventricular ejection function.

Antioxidants. Many studies (55-57) have shown that the most commonly used cardioprotective antioxidants (such as vitamin E, coenzyme Q10, glutathione and N-acetylcysteine) are not ideal. Flavonoids (especially monoHER), while having antioxidant effects, also have iron chelation and carbonyl reductase inhibition, showing advantages in preventing the cardiotoxicity of anthracyclines while not affecting their anti-tumor activity (10). The monoHER derivative frederine has a complete protective effect in the rat heart, and its effective dose is only $1 / 5$ of that of monoHER, although monoHER is more likely to be applied in the clinic (58).

\section{Treatment of cardiotoxicity}

In the early detection of anthracycline-induced cardiotoxicity symptoms, such as mild arrhythmia, atrial fibrillation, pericarditis, etc., anthracyclines should be discontinued by switching to a chemotherapy regimen without anthracyclines, and the necessary symptomatic treatment should be given.

When patients have symptoms of congestive heart failure, anti-heart failure treatments, such as converting enzyme inhibitors (ACEI), diuretics and $\beta$-blockers, can be given. A recent study showed that administration of ACEI or a combination of beta-blockers at the early detection of an anthracycline-induced cardiac insufficiency restored LVEF and reduced cardiac events (59).

\section{Future directions to avoid cardiotoxicity induced by anthracyclines}

There is no definitive and effective treatment for dose-dependent cardiotoxicity caused by anthracyclines. The cardiotoxicity of anthracyclines, in addition to factors related to the patient and the drug itself, is also associated with insufficient clinical attention. Monitoring and controlling heart toxicity caused by anthracyclines requires close cooperation between oncologists and cardiovascular physicians (18). It is necessary to establish a treatment monitoring specification for cardiotoxicity in breast cancer patients, and to fully assess the potential risks of treatment before it is started, in order to comprehensively understand patient heart function and tumor status, and to fully communicate with patients to minimize the risk of heart failure (Fig. 4).

In conclusion, further understanding of the mechanisms, monitoring methods, and preventive measures against cardiotoxicity caused by anthracyclines is of great significance for the early prevention of the cardiotoxicity of anthracyclines.

\section{Conclusion}

The current research on anthracycline-induced cardiotoxicity is still unclear and there is no effective prevention method. Anthracyclines are one of the most important drugs in the treatment of breast cancer. Therefore, while using these drugs, the risk factors associated with cardiotoxicity should be considered, cardiac function should be accurately measured and the cumulative dosage should be limited; otherwise, a new anthracycline alternative or liposome may be used to reduce anthracycline-induced cardiotoxicity. The clinician should develop a rational chemotherapy regimen based on individual differences and general conditions, and closely monitor the cardiotoxicity response induced by chemotherapeutic agents, in combination with various other examinations. Prevention of chronic cardiotoxicity is difficult, but regimens for the administration of the anthracyclines using prolonged infusion carry a lower risk and new liposomal preparations of doxorubicin offer less cardiotoxicity than traditional doxorubicin. The cardiac protectant dexrazoxane is effective in some clinical settings.

The treatment of anthracycline-induced heart failure is not well studied; it is likely that treatment should be similar to that for other types of heart failure.

\section{Acknowledgements}

Not applicable.

\section{Funding}

The present study was supported by The Scientific Research Foundation for the Returned Overseas Chinese Scholars, State Education Ministry (grant no. 2015-311), The Shanghai Health and Family Planning Commission Project (grant nos. 20134298, 201640253 and 201640226), The Shanghai Health and Family Planning Commission Fund for Qing Nian Yi Shi Training Project (grant no. 2014118), The Shanghai Yangpu District Science and Technology Commission Project (grant nos. 2016-2017, YP17ZM02 and 2017106), the Shanghai Yangpu District Health and Family Planning Commission Project (grant nos. 2011-2013 and 2016-2017, YP17ZM02, 2017106), the Shanghai Yangpu District Health and Family Planning Commission Fund for Hao Yi Shi Training Project (grant no. 201742), The Science Program from Yangpu Center Hospital (grant no. SE1201746), The Natural Science Foundation of Shanghai (grant no. 18ZR1436000) and The Fundamental Research Funds for the Central Universities (grant no. 22120180286).

\section{Availability of data and materials}

The datasets used and/or analyzed during the present study are available from the corresponding author on reasonable request.

\section{Authors' contributions}

FC reviewed the published literature, wrote the manuscript, reviewed and edited the final manuscript. MAFL reviewed the published literature, wrote sections of the manuscript and the reference section, and edited the final manuscript. XL 
reviewed the published literature, wrote and edited the reference section, and edited the final manuscript. MW reviewed the published literature, reviewed and edited the final manuscript. LC, CC and EB reviewed the published literature, wrote the manuscript, reviewed and edited the final manuscript. All authors read and approved the final version of the manuscript.

\section{Ethics approval and consent to participate}

Not applicable.

\section{Patient consent for publication}

Not applicable.

\section{Competing interests}

The authors declare that they have no competing interests.

\section{References}

1. Albini A, Pennesi G, Donatelli F, Cammarota R, De Flora S and Noonan DM: Cardiotoxicity of anticancer drugs: The need for cardio-oncology and cardio-oncological prevention. J Natl Cancer Inst 102: 14-25, 2010.

2. SEER Cancer Stat Facts: Female Breast Cancer. National Cancer Institute, Bethesda, MD, 2016. http://seer.cancer.gov/statfacts/html/breast.html. Accessed April 2019.

3. Canadian Cancer Society's Advisory Committee on Cancer Statistics: Canadian cancer statistics 2016. Canadian Cancer Society, Toronto, ON, 2016.

4. Cai FF, Kohler C, Zhang B, Wang MH, Chen WJ and Zhong XY: Epigenetic therapy for breast cancer. Int J Mol Sci 12: 4465-4487, 2011.

5. Siegel R, Naishadham D and Jemal A: Cancer statistics, 2012. CA Cancer J Clin 62: 10-29, 2012.

6. Alvarez RH: Present and future evolution of advanced breast cancer therapy. Breast Cancer Res 12 (Suppl 2): S1, 2010.

7. Zhang S, Liu X, Bawa-Khalfe T, Lu LS, Lyu YL, Liu LF and Yeh ET: Identification of the molecular basis of doxorubicin-induced cardiotoxicity. Nat Med 18: 1639-1642, 2012.

8. Mitry MA and Edwards JG: Doxorubicin induced heart failure: Phenotype and molecular mechanisms. Int J Cardiol Heart Vasc 10: 17-24, 2016.

9. Tocchetti CG, Cadeddu C, Di Lisi D, Femminò S, Madonna R, Mele D, Monte I, Novo G, Penna C, Pepe A, et al: From molecular mechanisms to clinical management of antineoplastic drug-induced cardiovascular toxicity: A translational overview. Antioxid Redox Signal: May 15, 2017 (Epub ahead of print).

10. Kaiserová H, Simůnek T, van der Vijgh WJ, Bast A and Kvasnicková E: Flavonoids as protectors against doxorubicin cardiotoxicity: Role of iron chelation, antioxidant activity and inhibition of carbonyl reductase. Biochim Biophys Acta 1772: 1065-1074, 2007.

11. Stewart DJ, Grewaal D, Green RM, Mikhael N, Goel R, Montpetit VA and Redmond MD: Concentrations of doxorubicin and its metabolites in human autopsy heart and other tissues. Anticancer Res 13: 1945-1952, 1993.

12. Mordente A, Meucci E, Silvestrini A, Martorana GE and Giardina B: New developments in anthracycline-induced cardiotoxicity. Curr Med Chem 16: 1656-1672, 2009.

13. Sanguinetti MC and Tristani-Firouzi M: hERG potassium channels and cardiac arrhythmia. Nature 440: 463-469, 2006.

14. Vandenberg JI, Perry MD, Perrin MJ, Mann SA, Ke Y and Hill AP: hERG $\mathrm{K}(+)$ channels: Structure, function, and clinical significance. Physiol Rev 92: 1393-1478, 2012.

15. Jehle J, Schweizer PA, Katus HA and Thomas D: Novel roles for hERG K(+) channels in cell proliferation and apoptosis. Cell Death Dis 2: e193, 2011

16. Curran ME, Splawski I, Timothy KW, Vincent GM, Green ED and Keating MT: A molecular basis for cardiac arrhythmia: HERG mutations cause long QT syndrome. Cell 80: 795-803, 1995.
17. Jaworski C, Mariani JA, Wheeler G and Kaye DM: Cardiac complications of thoracic irradiation. J Am Coll Cardiol 61: 2319-2328, 2013.

18. Jain D, Russell RR, Schwartz RG, Panjrath GS and Aronow W: Cardiac complications of cancer therapy: Pathophysiology, identification, prevention, treatment, and future directions. Curr Cardiol Rep 19: 36, 2017.

19. Wang G and Liu S: Research progress in cardiotoxicity of antitumor chemotherapy drugs. J Oncol 21: 1010-1014, 2015.

20. Manrique CR, Park M, Tiwari N, Plana JC and Garcia MJ: Diagnostic strategies for early recognition of cancer therapeutics-related cardiac dysfunction. Clin Med Insights Cardiol 11 $1179546817697983,2017$.

21. Von Hoff DD, Layard MW, Basa P, Davis HL Jr, Von Hoff AL, Rozencweig $\mathrm{M}$ and Muggia FM: Risk factors for doxorubicin-induced congestive heart failure. Ann Intern Med 91: 710-717, 1979.

22. Ryberg M, Nielsen D, Cortese G, Nielsen G and Andersen PK: New insight into epirubicin cardiac toxicity: Competing risks analysis of 1097 breast cancer patients. J Natl Cancer Inst 100: 1058-1067, 2008

23. Swain SM, Whaley FS and Ewer MS: Congestive heart failure in patients treated with doxorubicin: A retrospective analysis of three trials. Cancer 97: 2869-2879, 2003.

24. Tromp J, Steggink LC, Van Veldhuisen DJ, Gietema JA and van der Meer P: Cardio-oncology: Progress in diagnosis and treatment of cardiac dysfunction. Clin Pharmacol Ther 101: 481-490, 2017.

25. Hortobagyi GN, Frye D, Buzdar AU, Ewer MS, Fraschini G, Hug V, Ames F, Montague E, Carrasco CH, Mackay B, et al: Decreased cardiac toxicity of doxorubicin administered by continuous intravenous infusion in combination chemotherapy for metastatic breast carcinoma. Cancer 63: 37-45, 1989.

26. EBCTCG (Early Breast Cancer Trialists' Collaborative Group): Effect of radiotherapy after mastectomy and axillary surgery on 10-year recurrence and 20 -year breast cancer mortality: Meta-analysis of individual patient data for 8135 women in 22 randomised trials. Lancet 383: 2127-2135, 2014.

27. Pein F, Sakiroglu O, Dahan M,Lebidois J, Merlet P, Shamsaldin A, Villain E, de Vathaire F, Sidi D and Hartmann O: Cardiac abnormalities 15 years and more after Adriamycin therapy in 229 childhood survivors of a solid tumors at the Institute Gustave Roussy. Brit J Cancer 91: 37-44, 2004.

28. Schmitz KH, Prosnitz RG, Schwartz AL and Carver JR Prospective surveillance and management of cardiac toxicity and health in breast cancer survivors. Cancer 118 (Suppl 8): S2270-S2276, 2012.

29. Lang RM, Badano LP, Mor-Avi V, Afilalo J, Armstrong A, Ernande L, Flachskampf FA, Foster E, Goldstein SA, Kuznetsova T, et al: Recommendations for cardiac chamber quantification by echocardiography in adults: An update from the American society of echocardiography and the European association of cardiovascular imaging. J Am Soc Echocardiogr 28: 1-39.e14, 2015.

30. Tassan-Mangina S, Codorean D, Metivier M, Costa B Himberlin C, Jouannaud C, Blaise AM, Elaerts J and Nazeyrollas P: Tissue Doppler imaging and conventional echocardiography after anthracycline treatment in adults: Early and late alterations of left ventricular function during a prospective study. Eur J Echocardiogr 7: 141-146, 2006.

31. Jurcut R, Wildiers H, Ganame J, D'hooge J, De Backer J, Denys H, Paridaens R, Rademakers F and Voigt JU: Strain rate imaging detects early cardiac effects of pegylated liposomal doxorubicin as adjuvant therapy in elderly patients with breast cancer. J Am Soc Echocardiogr 21: 1283-1289, 2008.

32. Mitani I, Jain D, Joska TM, Burtness B and Zaret BL: Doxorubicin cardiotoxicity: Prevention of congestive heart failure with serial cardiac function monitoring with equilibrium radionuclide angio-cardiography in the current era. J Nucl Cardiol 10: 132-139, 2003.

33. van Royen N, Jaffe CC, Krumholz HM, Johnson KM, Lynch PJ, Natale D, Atkinson P, Deman P and Wackers FJ: Comparison and reproducibility of visual echocardiographic and quantitative radionuclide left ventricular ejection fractions. Am J Cardiol 77: $843-850,1996$

34. Armstrong AC, Gidding S, Gjesdal O, Wu C, Bluemke DA and Lima JA: LV mass assessed by echocardiography and CMR, cardiovascular outcomes, and medical practice. JACC Cardiovasc Imaging 5: 837-848, 2012. 
35. Ng R, Better N and Green MD: Anticancer agents and cardiotoxicity. Semin Oncol 33: 2-14, 2006.

36. McGowan JV, Chung R, Maulik A, Piotrowska I, Walker JM and Yellon DM: Anthracycline chemotherapy and cardiotoxicity. Cardiovasc Drugs Ther 31: 63-75, 2017.

37. Herman EH, Zhang J, Lipshultz SE, Rifai N, Chadwick D, Takeda K, Yu ZX and Ferrans VJ: Correlation between serum levels of cardiac troponin-T and the severity of the chronic cardiomyopathy induced by doxorubicin. J Clin Oncol 17: 2237-2243, 1999

38. Lipshultz SE, Rifai N, Sallan SE, Lipsitz SR, Dalton V, Sacks DB and Ottlinger ME: Predictive value of cardiac troponin $T$ in pedriatic patirnts at risk for myocardial injury. Circulation 96 : 2641-2648, 1997.

39. Porcel JM: Utilization of B-type natriuretic peptide and NT-proBNP in the diagnosis of pleural effusions due to heart failure. Curr Opin Pulm Med 17: 215-219, 2011.

40. Zhang N, Zheng L, Zheng J, et al: Significance of N-terminal pro-brain natriuretic peptide in early monitoring of anthracycline chemotherapy in elderly patients with breast cancer. Chin J Gerontol 6: 2931-2933, 2014.

41. Angelis AD, Cappetta D, Berrino L and Urbanek K: Doxorubicin cardiotoxicity: Multiple targets and translational perspectives. IntechOpen. https://www.intechopen.com/books/cardiotoxicity/ doxorubicin-cardiotoxicity-multiple-targets-and-translationalperspectives. Accessed November 14, 2008.

42. De Angelis A, Urbanek K, Cappetta D, Piegari E, Pia Ciuffreda L, Rivellino A, Russo R, Esposito G, Rossi F and Berrino L: Doxorubicin cardiotoxicity and target cells: A broader perspective. Cardiooncology 2: 2, 2016.

43. van Dalen EC, van der Pal HJ, Caron HN and Kremer LC: Different dosage schedules for reducing cardiotoxicity in cancer patients receiving anthracycline chemotherapy. Cochrane Database Syst Rev: CD005008, 2009.

44. Torti FM, Bristow MR, Howes AE, Aston D, Stockdale FE, Carter SK, Kohler M, Brown BW Jr and Billingham ME: Reduced cardiotoxicity of doxorubicin delivered on a weekly schedule: Assessment by endomyocardial biopsy. Ann Intern Med 99: 745-749, 1983

45. Koene RJ, Prizment AE, Blaes A and Konety SH: Shared risk factors in cardiovascular disease and cancer. Circulation 133 1104-1114, 2016

46. Harris L, Batist G, Belt R, Rovira D, Navari R, Azarnia N, Welles L and Winer E; TLC D-99 Study Group: Liposome-encapsulated doxorubicin compared with conventional doxorubicin in a randomized multicenter trial as first-line therapy of metastastic breast carcinoma. Cancer 94 25-36, 2002.

47. Minotti G, Menna P, Salvatorelli E, Cairo G and Gianni L: Anthracyclines: Molecular advances and pharmacologic developments in antitumor activity and cardiotoxicity. Pharmacol Rev 56: 185-229, 2004.

48. Van Dalen EC, Caron HN, Dickinson HO and Kremer LC: Cardioprotective interventions for cancer patients receiving anthracyclines. Cochrane Database Syst Rev: CD003917, 2008.
49. Che FF, Liu Y and Xu CG: Study on the effect and mechanism of dextromethionine on cardiotoxicity induced by doxorubicin. J Sichuan Univ 41: 24-28, 2010.

50. Gao X, Han Z and Du X: Observation of the effects of dextromethine on cardiotoxicity induced by epirubicin. Chin J Cancer Prev Treat 17: 296-298, 2010.

51. Professional Committee of Breast Cancer of China Anti-Cancer Association. Basic principles of chemotherapy for recurrent and metastatic breast cancer. Chin J Med 9: 73-75, 2011.

52. Sacco G, Bigioni M, Evangelista S, Goso C, Manzini S and Maggi CA: Cardioprotection effects of zofenopril, a new angiotension-converting enzyme inhibitor, on doxorubicin-induced cardiotoxicity in the rat. Eur J Pharmacol 414: 71-78, 2001.

53. Sacco G, Mario B, Lopez G, Evangelista S, Manzini S and Maggi CA: ACE inhibition and protection from doxorubicin-induced cardiotoxicity in the rat. Vascul Pharmacol 50: 166-170, 2009.

54. Kalay N, Basar E, Ozdogru I, Er O, Cetinkaya Y, Dogan A, Inanc T, Oguzhan A, Eryol NK, Topsakal R and Ergin A: Protective effects of carvedilol against anthracycline-induced cardiomyopathy. J Am Coll Cardiol 48: 2258-2262, 2006.

55. Elbaky NAA, El-Orabi NF, Fadda LM, Abd-Elkader OH and Ali HM: Role of N-acetylcysteine and coenzyme Q10 in the amelioration of myocardial energy expenditure and oxidative stress, induced by carbon tetrachloride intoxication in rats. Dose-Response 16: 1559325818790158, 2018.

56. Baker WL, Anglade MW, Baker EL, White CM, Kluger J and Coleman CI: Use of $\mathrm{N}$-acetylcysteine to reduce post-cardiothoracic surgery complications: A meta-analysis. Eur J Cardiothorac Surg 35: 521-527, 2009.

57. Kumar A, Kaur H, Devi P and Mohan V: Role of coenzyme Q10 (CoQ10) in cardiac disease, hypertension, and meniere-like syndrome. Pharmacol Ther 124: 259-268, 2009.

58. van Acker FA, Boven E, Kramer K, Haenen GR, Bast A and van der Vijgh WJ: Frederine, a new and promising protector against doxorubicin-induced cardiotoxicity. Clin Cancer Res 7: 1378-1384, 2001.

59. Mehta LS, Watson KE, Barac A, Beckie TM, Bittner V, Cruz-Flores S, Dent S, Kondapalli L, Ky B, Okwuosa T, et al: Cardiovascular disease and breast cancer: Where these entities intersect: A scientific statement from the American heart association. Circulation 137: e30-e66, 2018.

60. Khouri MG, Douglas PS, Mackey JR, Martin M, Scott JM, Scherrer-Crosbie M and Jones LW: Cancer therapy-induced cardiac toxicity in early breast cancer: Addressing the unresolved issues. Circulation 126: 2749-2763, 2012.

This work is licensed under a Creative Commons Attribution-NonCommercial-NoDerivatives 4.0 International (CC BY-NC-ND 4.0) License. 